\title{
A neonate with poor weight gain and hyperkalemia: Questions
}

\author{
Chiung-Chen Liu ${ }^{1} \cdot$ Shih-Hua Lin ${ }^{2} \cdot$ Chih-Chien Sung ${ }^{2,3} \cdot$ Chien-Ming Lin ${ }^{1,3}$
}

Received: 1 September 2015 /Revised: 8 November 2015 / Accepted: 10 November 2015 / Published online: 1 December 2015

(C) IPNA 2015

Keywords Hyperkalemia · Hyponatremia · Jaundice

A full-term female neonate who had experienced no birth asphyxia was admitted to our hospital at 12 days of life (D12) due to progressive yellowish skin discoloration of the face, trunk, and all limb regions beginning on D5, followed on subsequent days by frequent vomiting with persistent jaundice. Her birth weight was $2650 \mathrm{gm}$ (25-50th percentile) and body length was $46 \mathrm{~cm}$ (10-25th percentile). Family history was unremarkable. She was breastfed exclusively after birth, with adequate changing of diapers. Newborn screening for 17-hydroxyprogesterone and glucose-6-phosphate dehydrogenase was normal.

On admittance, her weight was 2468 gm (body weight loss of $6.8 \%$ ), blood pressure was $58 / 33 \mathrm{mmHg}$, heart rate was 188 beats/min, and respiratory rate was 38 breaths $/ \mathrm{min}$. Dry skin turgor and icterus were noted. The female external genitalia were normal without hyperpigmentation. Laboratory test results were as follows: serum total bilirubin, $21 \mathrm{mg} / \mathrm{dL}$ (normal value $<17 \mathrm{mg} / \mathrm{dL})$; direct bilirubin, $0.9 \mathrm{mg} / \mathrm{dL}(<2 \mathrm{mg} / \mathrm{dL})$; hemoglobin, $18.5 \mathrm{~g} / \mathrm{dL}(15-24 \mathrm{~g} / \mathrm{dL})$; aspartate transaminase,

The answers to these questions can be found at doi: 10.1007/s00467-0153281-5.

Chien-Ming Lin

ming.sandra@msa.hinet.net

1 Department of Pediatrics, Tri-Service General Hospital, National Defense Medical Center, No. 325, Cheng-Kung Road, Section 2, Neihu 114, Taipei, Taiwan, Republic of China

2 Division of Nephrology, Department of Medicine, National Defense Medical Center, Tri-Service General Hospital, Taipei, Taiwan

3 Graduate Institute of Medical Sciences, National Defense Medical Center, Taipei, Taiwan
$33 \mathrm{U} / \mathrm{L}(22-71 \mathrm{U} / \mathrm{L})$; alanine transaminase, $5 \mathrm{U} / \mathrm{L}(10-40 \mathrm{U} / \mathrm{L})$; sodium $(\mathrm{Na}), 131 \mathrm{mmol} / \mathrm{L}(133-146 \mathrm{mmol} / \mathrm{L})$; potassium $(\mathrm{K})$, $6.6 \mathrm{mmol} / \mathrm{L}(3.4-6.0 \mathrm{mmol} / \mathrm{L})$; chloride $(\mathrm{Cl}), 95 \mathrm{mmol} / \mathrm{L}(97-$ $110 \mathrm{mmol} / \mathrm{L}$ ). Despite treatment with intensive phototherapy and sufficient intravenous fluid supplementation, electrolyte abnormalities persisted on D13 ( Na $130 \mathrm{mmol} / \mathrm{L}, \mathrm{K}$ $6.0 \mathrm{mmol} / \mathrm{L}, \mathrm{Cl} 97 \mathrm{mmol} / \mathrm{L}$ ), although jaundice had improved (total bilirubin $13.9 \mathrm{mg} / \mathrm{dL}$ ).

Laboratory test results at $24 \mathrm{~h}$ after discontinuation of the medical intervention were as follows: blood urea level, $12 \mathrm{mg} /$ $\mathrm{dL}(3-12 \mathrm{mg} / \mathrm{dL})$; creatine, $0.5 \mathrm{mg} / \mathrm{dL}(0.34-0.45 \mathrm{mg} / \mathrm{dL}) ; \mathrm{Na}$, $126 \mathrm{mmol} / \mathrm{L} ; \mathrm{K}, 7.2 \mathrm{mmol} / \mathrm{L} ; \mathrm{Cl}, 94 \mathrm{mmol} / \mathrm{L}$; glucose, $107 \mathrm{mg} /$ dL (50-90 mg/dL); serum osmolality, $268 \mathrm{mOsm} / \mathrm{kgH}_{2} \mathrm{O}(275$ $295 \mathrm{mOsm} / \mathrm{kgH}_{2} \mathrm{O}$ ); cortisol, $23.49 \mathrm{ug} / \mathrm{dL}$ (1-24 ug/dL); plasma renin activity, $>50 \mathrm{ng} / \mathrm{mL} / \mathrm{h}(0.5-4.0 \mathrm{ng} / \mathrm{mL} / \mathrm{h})$; aldosterone lev$\mathrm{el},>25,000 \mathrm{pg} / \mathrm{mL}(50-1320 \mathrm{pg} / \mathrm{mL})$. Vein blood gas analysis revealed the following: $\mathrm{pH}, 7.405$ (7.32-7.45); partial pressure carbon dioxide $\left(\mathrm{PCO}_{2}\right), 28.9 \mathrm{mmHg}(41-51 \mathrm{mmHg})$; partial pressure oxygen $\left(\mathrm{PO}_{2}\right), 63.8 \mathrm{mmHg}(20-49 \mathrm{mmHg})$; bicarbonate $\left(\mathrm{HCO}_{3}\right), 17.7 \mathrm{mmol} / \mathrm{L}(24-28 \mathrm{mmol} / \mathrm{L})$; base excess $(\mathrm{BE})$, $-5.3 \mathrm{mmol} / \mathrm{L}$ ( -10 to $-2 \mathrm{mmol} / \mathrm{L}$ ). Spot urine sampling showed $\mathrm{Na}$ at $47 \mathrm{mmol} / \mathrm{L}, \mathrm{K}$ at $27.9 \mathrm{mmol} / \mathrm{L}, \mathrm{Cl}$ at $63 \mathrm{mmol} / \mathrm{L}$, and osmolality at $176 \mathrm{mOsm} / \mathrm{kgH}_{2} \mathrm{O}$. Urinalysis and abdominal sonography were normal.

\section{Compliance of ethical standards}

Conflicts of interest The authors declare that they have no conflict of interest.

\section{Questions \\ 1. What is your diagnostic approach to hyperkalemia? \\ 2. What is the diagnosis? \\ 3. How should this patient be managed?}

